\title{
THEORY OF INTERSECTION OF TWO PLANE CURVES
}

\section{CHUAN-CHIH HSIUNG}

1. Introduction. This paper is concerned with the study of the projective differential geometry of two plane curves intersecting at an ordinary point. The method used is similar to the one given by the author ${ }^{1}$ in a previous paper.

In $\$ 2$ we show the existence of two projective invariants $I, J$ determined respectively by the fourth order of the two curves at the point of intersection.

In $\$ 3$ by introducing a certain covariant triangle of reference associated to the two curves at the point of intersection, we reduce the invariants $I, J$ to simpler form and give them geometrical characterizations.

Finally, in $\$ 4$ a covariant point is suitably chosen for the unit point of the coordinate system in order to obtain the canonical power series expansions of the two curves at the point of intersection. With reference to the vanishing or nonvanishing of the two invariants $I, J$ we have four different types of expansions. The absolute invariants in the expansions of each type are interpreted geometrically in terms of certain double ratios.

2. Derivation of invariants. Let us establish a projective coordinate system in a plane, in which a point has nonhomogeneous coordinates $x, y$ and homogeneous coordinates $x_{1}, x_{2}, x_{3}$, connected by the relations $x=x_{2} / x_{1}, y=x_{3} / x_{1}$. The context will show in any instance which coordinates are being used. Then let us consider two curves $C, \bar{C}$ in the plane which intersect at an ordinary point $O$ with distinct tangents $t, \bar{t}$. If we choose the point $O$ to be the vertex $(1,0,0)$ of the triangle of reference, and the tangents $t, \bar{t}$ to be the sides $y=0, x=0$ of the triangle, then the power series expansions of the curves $C, \bar{C}$ in the neighborhood of the point $O$ may be written in the form

$$
\begin{cases}C: & y=a_{1} x^{2}+a_{2} x^{3}+a_{3} x^{4}+a_{4} x^{5}+\cdots, \\ \bar{C}: & x=\bar{a}_{1} y^{2}+\bar{a}_{2} y^{3}+\bar{a}_{3} y^{4}+\bar{a}_{4} y^{5}+\cdots, \quad a_{1} \bar{a}_{1} \neq 0 .\end{cases}
$$

Let us now make a most general transformation of coordinates which shall leave the point $O$ and the tangents $t, \bar{t}$ unchanged. This 1943.

Presented to the Society, September 13, 1943; received by the editors March 27,

${ }^{1}$ C. C. Hsiung, Projective differential geometry of a pair of plane curves, to appear in the Duke Mathematical Journal. 
transformation is expressed in terms of the nonhomogeneous coordinates by the equations

$$
\left\{\begin{array}{l}
x=B_{2} x^{*} /\left(1+A_{2} x^{*}+A_{3} y^{*}\right), \\
y=C_{3} y^{*} /\left(1+A_{2} x^{*}+A_{3} y^{*}\right) .
\end{array}\right.
$$

The effect of this transformation on equations (1) is to produce two other equations of the same form each of whose first three coefficients, indicated by stars, are given by the formulas

(3)

$$
\left\{\begin{array}{l}
C_{3} a_{1}^{*}=B_{2}^{2} a_{1}, \\
C_{3}\left(A_{2} a_{1}^{*}-a_{2}^{*}\right)=B_{2}^{2}\left(2 A_{2} a_{1}-B_{2} a_{2}\right), \\
C_{3}\left(A_{2}^{2} a_{1}^{*}-A_{3} a_{1}^{* 2}-A_{2} a_{2}^{*}+a_{3}^{*}\right)=B_{2}^{2}\left(3 A_{2}^{2} a_{1}-2 A_{3} a_{1} a_{1}^{*}\right. \\
B_{2} \bar{a}_{1}^{*}=C_{3}^{2} \bar{a}_{1}, \\
\left.B_{2}\left(A_{3} \bar{a}_{1}^{*}-\bar{a}_{2}^{*}\right)=C_{2}^{2} B_{2} a_{2}+B_{2}^{2} a_{3}\right) \\
B_{2}\left(A_{3}^{2} \bar{a}_{1}^{*}-A_{2} \bar{a}_{1}^{* 2}-A_{3} \bar{a}_{2}^{*}+C_{3} \bar{a}_{2}^{*}\right), \\
\quad-C_{3}^{2}\left(3 A_{3}^{2} \bar{a}_{1}-2 A_{2} \bar{a}_{1} \bar{a}_{1}^{*}\right. \\
\end{array}\right.
$$

Solving the first four of equations (3) for $A_{2}, A_{3}, B_{2}, C_{3}$ and subsituting the results in the fifth, it is easily seen that

$$
I=\left(1 / a_{1}\left(a_{1} \bar{a}_{1}^{2}\right)^{1 / 3}\right)\left(a_{1} \bar{a}_{2} / \bar{a}_{1}+a_{2}^{2} / a_{1}^{2}-a_{3} / a_{1}\right)
$$

is an invariant. In a way similar to the foregoing we have from the last one of equations (3) another invariant

$$
J=\left(1 / \bar{a}_{1}\left(a_{1}^{2} \bar{a}_{1}\right)^{1 / 3}\right)\left(\bar{a}_{1} a_{2} / a_{1}+\bar{a}_{2}^{2} / \bar{a}_{1}^{2}-\bar{a}_{3} / \bar{a}_{1}\right),
$$

which can be obtained also immediately by interchanging $a_{i}, \bar{a}_{i}$ $(i=1,2,3)$ from equation (4).

3. The canonical triangle and geometrical characterizations of the invariants $I, J$. By suitable choice of the other two vertices of the triangle of reference, expansions (1) can be simplified. For this purpose we consider the pencil of conics having contact of the third order with the curve $C$ at the point $O$. The equation of a general conic of this pencil is readily found to be

$$
a_{1}^{2} x^{2}+a_{2} x y-a_{1} y+k y^{2}=0,
$$

where $k$ is arbitrary. The coordinates of the pole $O_{1}$ of the tangent $\bar{t}$, $x=0$, with respect to this conic are 


$$
\left(a_{2}, a_{1}, 0\right) \text {. }
$$

Similarly, the pole $O_{2}$ of the tangent $t, y=0$, with respect to any conic having contact of the third order with the curve $\bar{C}$ at the point $O$ has the coordinates

$$
\left(\bar{a}_{2}, 0, \bar{a}_{1}\right) \text {. }
$$

It is convenient to call the points $O_{1}, O_{2}$ the associate points of the curves $C, \bar{C}$ at the point $O$. If these points be taken for the vertices $(0,1,0),(0,0,1)$ of the triangle of reference, then $a_{2}=\bar{a}_{2}=0$ and therefore equations (1) become

$$
\left\{\begin{array}{ll}
C: & y=a_{1} x^{2}+a_{3} x^{4}+a_{4} x^{5}+\cdots, \\
\bar{C}: & x=\bar{a}_{1} y^{2}+\bar{a}_{3} y^{4}+\bar{a}_{4} y^{5}+\cdots,
\end{array} \quad a_{1} \bar{a}_{1} \neq 0,\right.
$$

while the invariants $I, J$ take the simpler form

$$
\begin{aligned}
& I=-a_{3} / a_{1}^{2}\left(a_{1} \bar{a}_{1}^{2}\right)^{1 / 3}, \\
& J=-\bar{a}_{3} / \bar{a}_{1}^{2}\left(a_{1}^{2} \bar{a}_{1}\right)^{1 / 3} .
\end{aligned}
$$

Thus the coordinate system except the unit point has been determined, and the triangle $O O_{1} O_{2}$ will be called the canonical triangle of the curves $C, \bar{C}$ at the point $O$.

We are now in position to characterize geometrically the invariants $I, J$ by expressing them in terms of certain double ratios. To this end let us consider the conic which passes through the associate point $\mathrm{O}_{2}$ and has contact of the third order with the curve $C$ at the point $O$. Hereafter we shall call this conic the associate conic of the curve $C$ at the point $O$. From equation (6) its equation with respect to the canonical triangle

$$
a_{1} x^{2}-y=0
$$

follows immediately. Similarly, we obtain the equation of the associate conic of the curve $\bar{C}$ at the point $O$,

$$
\bar{a}_{1} y^{2}-x=0 \text {. }
$$

The two associate conics (12), (13) intersect, besides in $O$, in three points $P_{i}(i=1,2,3)$ with coordinates

$$
\left(\epsilon^{i} /\left(a_{1}^{2} \bar{a}_{1}\right)^{1 / 3}, \epsilon^{2 i} /\left(a_{1} \bar{a}_{1}^{2}\right)^{1 / 3}\right),
$$

where $\epsilon^{3}=1$. Let $P_{k}^{\prime}$ be the intersections of the lines $O P_{k}, P_{i} P_{j}$, where $i, j, k=1,2,3$ and are all distinct. It is evident that $P_{1}^{\prime} P_{2}^{\prime}$ is the 
unique real one among the three lines $P_{i}^{\prime} P_{j}^{\prime}$. Its equation may be easily found to be

$$
1-2\left(a_{1}^{2} \bar{a}_{1}\right)^{1 / 3} x-2\left(a_{1} \bar{a}_{1}^{2}\right)^{1 / 3} y=0,
$$

and we shall call it the real associate line of the curves $C, \bar{C}$ at the point $O$. This real associate line intersects the tangent $\bar{t}, x=0$, in the point $R$,

$$
\left(0,1 / 2\left(a_{1} \bar{a}_{1}^{2}\right)^{1 / 3}\right) .
$$

On the other hand we consider a general cubic curve which has contact of the fourth order with the curve $C$ at the point $O$ and a node at $O$ with $t, \bar{t}$ for nodal tangents. A simple calculation suffices to give the equation of this nodal cubic curve, namely,

$$
a_{1}^{8} x^{3}+a_{3} x y^{2}-a_{1}^{2} x y+k y^{3}=0,
$$

where $k$ is arbitrary. The line containing the three inflexions of the cubic (17) has the equation

$$
y=a_{1}^{2} / a_{3},
$$

which is intersected by the tangent $\bar{t}, x=0$, in the point $S$,

$$
\left(0, a_{1}^{2} / a_{3}\right) \text {. }
$$

From equations (10), (16), (19) it follows at once that the double ratio of the four points $O, O_{2}, R, S$ is equal to

$$
\left(O O_{2}, R S\right)=-I / 2 \text {. }
$$

Hence we obtain the following geometrical characterization of the invariant $I$.

Let two plane curves $C, \bar{C}$ intersect at an ordinary point $O$ with distinct tangents $t, \bar{t}$. Let $\mathrm{O}_{2}$ be the associate point of the curve $\bar{C}$ at the point $O$, and $C_{3}$ a general cubic curve which has contact of the fifth order with the curve $C$ at the point $O$ and a node at $O$ with $t, \tilde{t}$ for nodal tangents. If the tangent $\bar{t}$ intersects the real associate line of the curves $C, \bar{C}$ at $O$ in point $R$, and intersects the line containing the three inflexions of the nodal cubic curve $C_{3}$ in point $S$; then the invariant I associated with the point $O$ of the curves $C, \bar{C}$ is, except for sign, equal to two times the double ratio $\left(\mathrm{OO}_{2}, \mathrm{RS}\right)$.

The other invariant $J$ can be characterized geometrically in a similar manner.

4. Canonical power series expansions. In this section we shall com- 
plete the characterization of the coordinate system by suitably choosing a covariant point for the unit point, and obtain the canonical power series expansions of the curves $C, \bar{C}$ in the neighborhood of the point $O$. It will be convenient to discuss four cases with reference to the vanishing or nonvanishing of the two invariants $I, J$.

Case I. $I J \neq 0$. Here we can choose for the unit point the intersection of the line of inflexions (18) and the similar one

$$
x=\bar{a}_{1}^{2} / \bar{a}_{3},
$$

so that $a_{3}=a_{1}^{2}, \bar{a}_{3}=\bar{a}_{1}^{2}$. Thus we arrive at the canonical power series expansions of the curves $C, \bar{C}$ in the neighborhood of the point $O$,

$$
\left\{\begin{array}{ll}
C: & y=a_{1} x^{2}+a_{1}^{2} x^{4}+\cdots, \\
\bar{C}: & x=\bar{a}_{1} y^{2}+\bar{a}_{1}^{2} y^{4}+\cdots,
\end{array} \quad a_{1} \bar{a}_{1} \neq 0,\right.
$$

in which the coefficients $a_{1}, \bar{a}_{1}$ can be expressed in terms of the two invariants $I, J$, namely,

$$
a_{1}=-I / J^{2}, \quad \bar{a}_{1}=-J / I^{2} .
$$

Case II. $I=0, J \neq 0$. In this case we observe that in the pencil (17) of nodal cubic curves there is a unique one having contact of the fifth order with the curve $C$ at the point $O$. Making use of the first of equations (9) and noticing that $a_{3}=0$ we find at once the equation of this cubic, namely,

$$
a_{1}^{4} x^{3}+a_{4} y^{3}-a_{1}^{3} x y=0 .
$$

The join $m$ of the two real intersections of the cubic curve (24) and the associate conic (13) of the curve $\bar{C}$ at $O$ is given by the equation

$$
a_{1} a_{1}^{1 / 3} x+\left(a_{4}-a_{1}^{3} \bar{a}_{1}\right)^{1 / 3} y=0 .
$$

If the unit point of the coordinate system be taken at the intersection of the lines (21), (25), then $a_{4}=a_{1}^{3}\left(\bar{a}_{1}-a_{1}\right), \bar{a}_{3}=\bar{a}_{1}^{2}$, and therefore the canonical power series expansions of the curves $C, \bar{C}$ in the neighborhood of the point $O$ are reached:

$$
\left\{\begin{array}{ll}
C: & y=a_{1} x^{2}+a_{1}^{3}\left(\bar{a}_{1}-a_{1}\right) x^{5}+\cdots, \\
\bar{C}: & x=\bar{a}_{1} y^{2}+\bar{a}_{1}^{2} y^{4}+\cdots,
\end{array} \quad a_{1} \bar{a}_{1} \neq 0 .\right.
$$

Accordingly, the invariant $J$ becomes

$$
J=-1 /\left(a_{1}^{2} \bar{a}_{1}\right)^{1 / 3} .
$$

In $\$ 2$ we have shown that there are only two independent invari- 
ants determined by the neighborhoods up to and including the fourth order of the curves $C, \bar{C}$ at the point $O$, one of them vanishing now. In order to exhaust the two independent coefficients in the canonical expansions (26) it is sufficient for us to find another independent invariant of the curves $C, \bar{C}$ at the point $O$. For this purpose let $r$ be the join of the two real intersections of the two associate conics (12), (13), whose equation is found to be

$$
a_{1}^{1 / 3} x-a_{1}^{1 / 3} y=0 .
$$

From equations (25), (28) the following double ratio is immediate:

$$
\Delta \equiv(t \bar{t}, m r)=\left(\bar{a}_{1} / a_{1}\right)^{1 / 3} \text {. }
$$

Thus the two coefficients $a_{1}, \bar{a}_{1}$ in expansions (26) have been completely expressed in terms of the invariant $J$ and the double ratio $\Delta$, namely,

$$
a_{1}=-1 / J \Delta, \quad \bar{a}_{1}=-\Delta^{2} / J .
$$

Case III. $I \neq 0, J=0$. Similarly, with the rôles of the curves $C, \bar{C}$ in Case II interchanged we obtain the desired canonical power series expansions for this case,

$$
\begin{cases}C: & y=a_{1} x^{2}+a_{1}^{2} x^{4}+\cdots, \\ \bar{C}: & x=\bar{a}_{1} y^{2}+\bar{a}_{1}^{3}\left(a_{1}-\bar{a}_{1}\right) y^{5}+\cdots, \quad a_{1} \bar{a}_{1} \neq 0,\end{cases}
$$

in which the two coefficients $a_{1}, \bar{a}_{1}$ can be expressed also in terms of the invariant I and a double ratio similar to (29).

Case IV. $I=0, J=0$. It is useful for this case to consider the cubic curve which has contact of the fifth order with the curve $\bar{C}$ at the point $O$ and a node at $O$ with $t, \bar{t}$ for nodal tangents. The equation of this cubic can be written immediately by interchanging $x, y$ and $a_{i}, \bar{a}_{i}(i=1,4)$ from equation (24). The result is

$$
\bar{a}_{4} x^{3}+\bar{a}_{1}^{4} y^{3}-\bar{a}_{1}^{3} x y=0 .
$$

The line $n$ joining the associate point $O_{2}$ to the real intersection, other than $O$, of the cubic curve (32) and the associate conic (12) of the curve $C$ at $O$ is given by the equation

$$
x=\left(1 / a_{1} \bar{a}_{1}\right)\left(a_{1} \bar{a}_{1}^{2}-\bar{a}_{4} / \bar{a}_{1}\right)^{1 / 3} .
$$

If the unit point of the coordinate system be taken at the intersection of the lines (25), (33), then $a_{4}=a_{1}^{3}\left(\bar{a}_{1}-a_{1}\right), \bar{a}_{4}=a_{1} \bar{a}_{1}^{3}\left(1-a_{1}^{2} \bar{a}_{1}\right)$, and therefore we reach the required canonical power series expansions, 


$$
\begin{cases}C: & y=a_{1} x^{2}+a_{1}^{3}\left(\bar{a}_{1}-a_{1}\right) x^{5}+\cdots, \\ \bar{C}: & x=\bar{a}_{1} y^{2}+a_{1} \bar{a}_{1}^{3}\left(1-a_{1}^{2} \bar{a}_{1}\right) y^{5}+\cdots, \quad a_{1} \bar{a}_{1} \neq 0 .\end{cases}
$$

In order to interpret geometrically the coefficients in the canonical expansions (34), let us assume $s$ to be the line joining the associate point $\mathrm{O}_{2}$ to the real intersection, other than $O$, of the two associate conics (12), (13), whose equation is found to be

$$
x_{1}-\left(a_{1}^{2} \bar{a}_{1}\right)^{1 / 3} x_{2}=0 .
$$

From equations (33), (35) we obtain at once the following double ratio,

$$
D \equiv\left(\bar{t}, O_{1} O_{2}, n, s\right)=\left(a_{1}^{2} \bar{a}_{1}\right)^{1 / 3} .
$$

Thus the two coefficients $a_{1}, \bar{a}_{1}$ in expansions (34) have been completely expressed in terms of the two double ratios $\Delta, D$, namely,

$$
a_{1}=D / \Delta, \quad \bar{a}_{1}=D \Delta^{2} .
$$

Some of the results of this section are contained in the following summary.

Let two plane curves $C, \bar{C}$ intersect at an ordinary point $O$ with distinct tangents $t, \bar{t}$. The projective differential geometry of the curves $C, \bar{C}$ in the neighborhood of the point $O$ can be studied by means of expansions (22), (26), (31), (34) of four different types, all of whose coefficients are absolute invariants of the curves. The covariant coordinate system for each type of expansions has the following geometric description. The vertex $(1,0,0)$ of the triangle of reference is the point $O$ under consideration, and the other vertices $(0,1,0),(0,0,1)$ are the associate points $O_{1}, O_{2}$ of the curves $C, \bar{C}$ at the point $O^{\circ}$. Let $C_{3}\left(\bar{C}_{3}\right)$ be a general one of a pencil of cubic curves which have contact of the fourth order with the curve $C(\bar{C})$ at the point $O$ and a node at $O$ with $t, \bar{t}$ for nodal tangents, $K_{3}\left(\bar{K}_{3}\right)$ the unique cubic of this pencil having contact of the fifth order with the curve $C(\bar{C})$ at the point $O$, and $l(\bar{l})$ the line of inflexions of the nodal cubic curve $C_{3}\left(\bar{C}_{3}\right)$. Further, let $m(\bar{m})$ be the join of the two real intersections of the cubic $K_{3}\left(\bar{K}_{3}\right)$ with the associate conic $\bar{C}_{2}\left(C_{2}\right)$ of the curve $\bar{C}(C)$ at $O$, and $n$ the line joining the associate point $\mathrm{O}_{2}$ to the real intersection, other than $O$, of the cubic $\bar{K}_{3}$ with the associate conic $C_{2}$. Then the unit point of the covariant coordinate system for each type of expansions is respectively one of the intersections of the four pairs of lines: (i) $l$ and $\bar{l}$, (ii) $\bar{l}$ and $m$, (iii) $l$ and $\bar{m}$, and (iv) $m$ and $n$.

The National University of Chekiang 